\title{
ESTUDO COMPARATIVO DE ALGORITMO DE NEWTON VERSUS SISTEMAS IMUNOLÓGICOS ARTIFICIAIS E APLICAÇÃO EM MINIMIZAÇÃO DE FLUXO EM LINHAS DE TRANSMISSÃO
}

\author{
Marcelo A. Canavez ${ }^{(1)}$ (marcelocanavez2@gmail.com), Jéssica B. Oliveira ${ }^{(2)}$ \\ (jessicabonoto@yahoo.com.br), Leônidas C.de Resende ${ }^{(3)}$ (leonidas@ufsj.edu.br) \\ (1,2) Programa de Pós-Graduação em Engenharia Elétrica (UFSJ), Praça Frei Orlando, 170 - Centro, São João del-Rei - MG, Brasil \\ (3) Universidade Federal de São João del-Rei (UFSJ) - DEPEL - Praça Frei Orlando, 170 - Centro, São João del-Rei - MG, Brasil
}

RESUMO: Este artigo traz uma revisão sobre o algoritmo de otimização baseado em meta-heurística e inspirado em Sistemas Imunológicos Artificiais (AIS), através do Algoritmo de Seleção Clonal com Reconfiguração (CLONR). É apresentada também uma breve contextualização e equacionamento do Método de Newton, como método baseado em derivação. São apresentados três exemplos com o objetivo de se tornar um guia para desenvolvimento e resolução via Newton e via CLONR. Desta forma os resultados obtidos pelos dois algoritmos podem ser comparados em relação ao ponto ótimo e ao valor da função objetivo neste ponto. Esse trabalho apresenta entre as aplicações a minimização de fluxo de potência em uma linha de transmissão de um sistema de 3 barras, respeitando as limitações de tensão e potência do sistema e a não motorização do gerador. O Método CLONR se mostra fortemente ligado ao chute inicial e leva mais iterações que o Método de Newton para convergência. Porém, estas iterações são demasiadamente rápidas e podem resolver problemas complexos. Este método, assim como outros baseados em meta-heurística, não têm a obrigatoriedade de a função objetivo possuir derivadas de altas ordens, o que acontece com o Método de Newton e outros baseados em derivação.

PALAVRAS-CHAVE: Otimização, Sistema Imunológico Artificial, CLONR, Fluxo de Potência, Newton, CLONALG.

\section{INTRODUÇÃO}

O avanço constante na tecnologia e aumento na competitividade exigem projetos que busquem cada vez mais melhores soluções ou soluções otimizadas. A otimização consiste na obtenção de uma solução ou um conjunto de soluções ótimas para determinada função ou conjunto de funções, também conhecido como função objetivo, ou ainda, buscar a melhor solução dentre as alternativas disponíveis. É uma ferramenta de grande aplicabilidade que constitui uma vasta e atraente área do conhecimento, sendo importante não apenas para as engenharias e ciências exatas, mas também para as mais diversas áreas de biologia e tecnologia (Oliveira et. al, 2012). Desta forma, a pesquisa de algoritmos adequados para se chegar mais facilmente à solução de determinados grupos de problemas, passa a ser relevante. Existem diversos algoritmos de otimização destinados a tratar uma variada gama de problemas de engenharia (Silva, 1997).

O Método de Newton é um método clássico muito utilizado para obtenção de soluções de problemas de otimização. Ele depende somente das derivadas de primeira e segunda ordem e de alguns parâmetros de entrada para obter uma rápida convergência para os pontos críticos da função objetivo. Baseado na melhoria do Método do Gradiente considerando a expansão da série de Taylor 

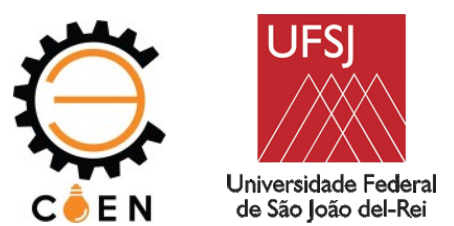

(Swann, 1969). Swann apresenta ainda outros métodos com características similares para otimização não linear.

Contudo, por dependerem da existência da derivada segunda, as abordagens do Método de Newton para programação linear e quadrática resultam em soluções fracas para problemas não convexos, descontínuos ou com soluções muito lineares, não sendo tão eficientes. O progresso próximo ao ponto crítico é também assegurado somente se a matriz de derivadas segundas, $G$ for definida positiva. Contudo, o método é eficiente na vizinhança de pontos críticos onde a função é análoga à quadrática e a matriz de derivada segunda é definida positiva. Longe do mínimo o progresso pode ser mais lento ou até levar a divergência. Além disso, o processo de derivação, montagem e inversão da matriz de derivadas segundas tende a consumir alto tempo computacional. Já as abordagens meta-heurísticas, podem ser introduzidas com objetivo de otimizar as funções objetivo escolhidas, fornecendo soluções globais (Swann, 1969; Ridzuan et. al, 2018).

Muitos problemas não podem ser otimizados pelo uso de métodos diferenciais, ou as soluções obtidas podem não ser ótimas, ou ainda podem levar muito tempo para a convergência. Os algoritmos meta-heurísticos são projetados para resolver de maneira aproximada estes problemas sem ter que aprofundar muito para adaptar a cada problema, tendo principais aplicações em indústrias, serviços e finanças e em áreas mais específicas, como processamento de sinais, projeto de filtros e controle de robôs (Liu et. al, 2007; Boussaid et. al, 2013). Essas técnicas se inspiram em comportamentos encontrados no mundo real para realizar uma busca eficiente na direção de uma solução de boa qualidade. Para isso, mecanismos que evoluem um conjunto inicial de possíveis soluções a uma maior aproximação da solução ótima (Seta et. ala, 2015).

Dentre estas técnicas, pode-se citar Busca Tabu, Algoritmo Genético (GA), Recozimento Simulado, Otimização por Enxame de Partículas (PSO) e Algoritmos baseado em Sistemas Imunológicos Artificiais (AIS). Contudo, o enfoque será dado ao método aplicado neste trabalho o AIS.

AIS é uma técnica de busca de soluções que emergiu na década de 1990 originada da habilidade de aprendizagem e reconhecimento de padrões do sistema imunológico humano, formado por circuitos de células, tecidos e órgãos que trabalham em conjunto para defender o corpo contra ataques de invasores. Este sistema protege o organismo de patógenos (micro-organismos nocivos como bactérias e vírus) sem o conhecimento anterior sobre os mesmos. Além disso, é distribuído, reativo e adaptativo, consistindo em uma variedade de interações de elementos celulares e moleculares ao longo do corpo. Tem uma grande capacidade de aprendizagem dinâmica e memória e apresenta um tipo evolutivo de resposta a elementos infecciosos externos, além de propriedades de diversidade, computação distribuída, tolerância de erro e monitoramento (Lau et. al, 2008; Boussaid et. al, 2013; Singh et. al, 2017; Tian et. al, 2018).

A busca imunológica tende a ser mais robusta que GA e PSO, porque começa a procurar ao longo de todo o espaço e se afasta da convergência prematura para mínimos locais (Singh et. al, 2017). Dentre os AIS, quatro se destacam: algoritmos de seleção negativa; circuitos imunológicos artificiais; teoria do perigo e os algoritmos de células dendríticas; algoritmos de seleção clonal (Boussaid et. al, 2013). Neste trabalho será utilizado o algoritmo CLONR, uma variação do Algoritmo de Seleção Clonal (CLONALG) e é apresentado na Seção Metodologia.

Algoritmos baseados no sistema imunológico possuem diversas aplicações voltadas ao sistema elétrico, entre elas Ferreira et. al (2015), Seta et. $\mathrm{al}^{\mathrm{a}}$ (2015), Seta et. $\mathrm{al}^{\mathrm{b}}$ (2015), Cossi et. al (2016), Singh et. al (2017), Navarro-Caceres (2018), Ridzuan et. al (2018), Shin et. al (2018), e ao 

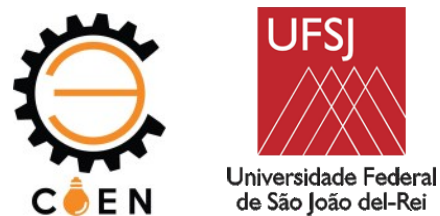

machine learning em Tian (2018). Este trabalho também aplica os métodos propostos a um exemplo voltado ao sistema elétrico.

A Seção Metodologia traz o equacionamento e procedimento para desenvolvimento do Método de Newton e do Método da AIS, baseado no algoritmo CLONR. Após, na Seção Aplicações estes são aplicados a três exemplos como forma de comparar o desempenho dos métodos em termos do número de iterações, valor das variáveis encontradas e valor da função objetivo otimizada.

\section{METODOLOGIA}

Primeiramente, antes da explicação de como são aplicados o Método de Newton e o da AIS, deve-se formular o problema:

Seja f: $R^{n} \rightarrow \mathfrak{R}$ uma função real de $n$ variáveis. O valor da função é escrito como $f(x)=f\left(x_{1}, x_{2}, \ldots\right.$, $x_{n}$ ) para $x_{i} \in R^{n}$. Esta é a função a ser minimizada, ou função objetivo. $x^{*} \in R^{n}$ é um mínimo global se $f\left(x^{*}\right)<f(x)$ para todo $x$ pertencente ao domínio (Zeidler, 2013).

Contudo, a análise do ponto que minimiza a função passa a ser mais complexa, a partir do momento em que são adicionadas restrições ao problema, como a restrição de igualdade na Equação 1 .

$$
\left\{\begin{array}{c}
\operatorname{Min} f(x) \\
\text { Sujeito a } g_{i}(x)=0
\end{array}\right.
$$

Para resolver o problema com restrições de igualdade, existe um método chamado de Método de Lagrange que afirma: ao buscar o máximo ou mínimo de uma função multivariável sujeita a uma ou mais funções de restrição, então essas funções devem ser multiplicadas por fatores indeterminados e acrescidas à função cujo extremo é procurado, buscando o máximo ou mínimo da soma resultante como se as variáveis fossem independentes. Desta forma têm-se o problema sem restrições: (De Klerk et. al, 2004; Saramago et. al, 2008). A Equação 2 mostra como é feito este procedimento.

$$
\operatorname{Min} L(x, \lambda)=f(x)+\sum_{j=1}^{m} \lambda_{j} g_{j}(x)
$$

Podem ser ainda adicionadas restrições de desigualdade como mostra a Equação 3.

$$
\left\{\begin{array}{c}
\operatorname{Min} L(x, \lambda) \\
\text { Sujeito } a h_{i}(x) \leq 0
\end{array}\right.
$$

De acordo com Kuhn-Tucker, esta restrição de desigualdade pode ser utilizada de forma a transformar $L$ em uma nova função irrestrita, transformando o problema na Equação 4 (Santos, 1998; De Klerk et. al, 2004; Saramago et. al, 2008; Matei et. al, 2015).

$$
\operatorname{Min} L(x, \lambda, \mu)=f(x)+\sum_{j=1}^{m} \lambda_{j} g_{j}(x)+\sum_{k=1}^{l} \mu_{k} h_{k}(x)
$$


Quando um ponto $x \in R^{n}$ satisfaz todas as restrições, diz-se que ele é viável e que o conjunto de todos os pontos viáveis é denominado região viável $\Gamma$ (Silva, 1997). Para que um ponto seja um ponto de mínimo deve cumprir 5 condições necessárias e suficientes, também sintetizadas nas Equações 5 a 8 (Santos, 1998; De Klerk et. al, 2004; Saramago et. al, 2008; Zeidler, 2013).

1) $x^{*}$ viável.

2) $\lambda g\left(x^{*}\right)=0, \lambda \leq 0$

3) $\mu h\left(x^{*}\right)=0, \mu \geq 0$

4): $L\left(x^{*}, \lambda^{*}, \mu^{*}\right)$ deve ser estacionário, ou seja, possuir gradiente nulo.

$$
\left\{\begin{array}{c}
\nabla_{x} L\left(x^{*}, \lambda^{*}, \mu^{*}\right)=0 \\
\nabla_{x} f(x)+\sum_{j=1}^{m} \nabla_{x} \lambda_{j} g_{j}(x)+\sum_{k=1}^{l} \nabla_{x} \mu_{k} h_{k}(x)=0
\end{array}\right.
$$

5): Cada função de restrição deve ser duplamente diferenciável e a matriz Hessiana deve ser definida positiva sob o espaço analisado, ou seja:

$$
\left\{\begin{array}{c}
\nabla_{x}^{2} L\left(x^{*}, \lambda^{*}, \mu^{*}\right) \neq 0 \\
\nabla_{x}^{2} f(x)+\sum_{j=1}^{m} \nabla_{x}^{2} \lambda_{j} g_{j}(x)+\sum_{k=1}^{l} \nabla_{x}^{2} \mu_{k} h_{k}(x) \neq 0
\end{array}\right.
$$

Observando as condições 4) e 5), observa-se que os métodos diferenciáveis são extremamente sensíveis à derivação da função objetivo e das funções de restrições. Caso alguma das condições não seja atendida não há garantia de convergência para a resposta otimizada procurada. $\mathrm{O}$ método diferencial utilizado neste trabalho é o Método de Newton, descrito pelas Equações 9 a 13 e possui a seguinte formulação (Swann, 1969):

Seja $g$ o vetor de derivadas parciais de primeira ordem da função, $G$ é a matriz de derivadas parciais de segunda ordem e $\delta$ representa a distancia entre o mínimo e o ponto atual no processo de busca da solução.

$$
\begin{gathered}
g_{i}=\frac{\partial f}{\partial x_{i}}, \quad G_{i j}=\frac{\partial^{2} f}{\partial x_{i} \partial x_{j}}, \quad i, j=1,2, \ldots, n . \\
f(x)=f\left(x^{*}+\delta\right) \approx f\left(x^{*}\right)+\delta^{\prime} g+\frac{1}{2} \delta^{\prime} G \delta
\end{gathered}
$$
satisfazer:

No mínimo, as derivadas parciais são iguais a zero, então o gradiente no ponto $x$ deve

$$
g=G \delta
$$



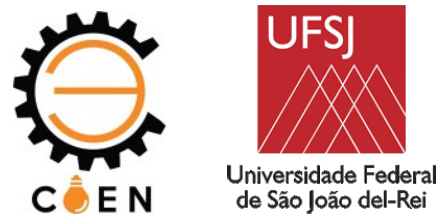

Basta mover agora $x^{*}$ na direção do mínimo em $\delta$, que representa a solução das equações lineares simultâneas. Desta forma, pode-se escrever:

$$
x^{*}=x-G^{-1} g
$$

Então, se as funções são quadráticas, o mínimo pode ser localizado aplicando a equação anterior, com $g$ avaliado nos pontos $x$, e $G$ avaliado no mínimo, neste caso o método é dito quadraticamente convergente.

Quando a função não é quadrática, uma abordagem iterativa deve ser adotada em que $g$ e $G$ são calculados no ponto $x_{i}$ e a aproximação do mínimo é obtida usando:

$$
x^{i+1}=x^{i}-\left(G^{i}\right)^{-1} g^{i}
$$

No caso multivariável, como é o problema que possui restrições, a matriz de derivadas parciais de primeira e segunda ordem deve ter os termos de derivadas em relação à $\mu, \lambda, x$ e suas combinações, prosseguindo de maneira análoga. A matriz $G$ que conterá derivadas parciais de segunda ordem continuará a ser invertida somente uma vez para cada iteração. Uma vez explicado como o método de Newton será aplicado ao problema de otimização, faz-se necessário explicar como o algoritmo de busca imunológica trabalhará com as restrições e sua formulação.

Para minimizar uma função $f(x)$, sujeita a restrições, adota-se o seguinte procedimento para busca imunológica: Minimiza-se a função objetivo, como uma função sem restrições, mas introduzindo penalidades para limitar a violação das restrições. Assim, cria-se uma nova função objetivo, denominada função pseudo-objetivo. O ótimo do projeto restrito é obtido através da solução sequencial de vários problemas sem restrição. Estes métodos são conhecidos também como SUMT (Sequential Unconstrained Minimization Techniques) (Saramago, 2008; Sampaio, 2014).

Desta forma, o problema original transforma-se num problema de um único objetivo com as restrições originais inseridas e serve para obter uma aproximação da fronteira de maneira mais simples e eficiente. Assumindo um peso para cada restrição violada, pode-se reescrever a Equação 14 partir da Equação 4:

$$
\operatorname{Min} L(x, \lambda, \mu)=f(x)+\sum_{j=1}^{m} w_{j} g_{j}(x)+\sum_{k=1}^{l} w_{k} h_{k}(x)
$$

Em que $w_{i}=0$, se as restrições não são violadas e $w_{i}=$ número elevado se elas são violadas. Desta forma quando as restrições são violadas o valor da função se torna elevado, deixando de ser atrativo para minimização. Por simplicidade, nos exemplos trabalhados que envolvem restrições, os pesos foram adotados como sendo iguais. Sendo $w=50$, na segunda aplicação e $w=100$ na terceira, de forma a não se impossibilitar a busca de soluções próximas aos pontos que não atendam às restrições. Uma vez especificadas as premissas de resolução do problema a ser especificado, define-se agora o funcionamento do algoritmo de busca imunológica.

O algoritmo CLONALG ou CLONR pode ser resumido como se segue: os linfócitos são produzidos aleatoriamente na medula óssea, formando células, que produzem anticorpos. Cada anticorpo apresenta familiaridade com determinado antígeno invasor e através da seleção das que 
apresentam maior familiaridade, com consequente clonagem e mutação melhora-se a afinidade destes anticorpos com o antígeno. Esse processo é dinâmico com a medula continuando a produzir células e comparação entre as novas células e as "melhoradas" buscando a cada iteração um melhor anticorpo, comparando inclusive com os já clonados. Através desta atuação conjunta de seleção clonal das células com maior afinidade e hipermutação somática, a afinidade dos novos anticorpos produzidos é novamente analisada e repete-se o processo até que a convergência seja obtida. Assim, têm-se uma maior exploração das soluções locais e das soluções aleatórias em algoritmos de otimização, baseado na avaliação dos anticorpos, com subsequente clonagem e mutação. $\mathrm{O}$ critério de parada é quando o número de iterações máximo é atingido, ou até que ocorra um número de iteração especificado sem melhora na solução, conhecido como critério de estagnação (Boussaid et. al, 2013; Ferreira et. al, 2015; Seta $^{\text {a }}$ et. al, 2015; Seta ${ }^{\mathrm{b}}$ et. al, 2015; Singh et. al, 2017; Tian et. al, 2018; Navarro-Caceres, 2018;).

A Figura abaixo mostra como funciona o algoritmo CLONR utilizado neste trabalho De Oliveira et. al (2014). Este procedimento deve ser realizado para cada variável do problema, o que pode ser feito utilizando os mesmos loops, com economia de tempo de simulação.

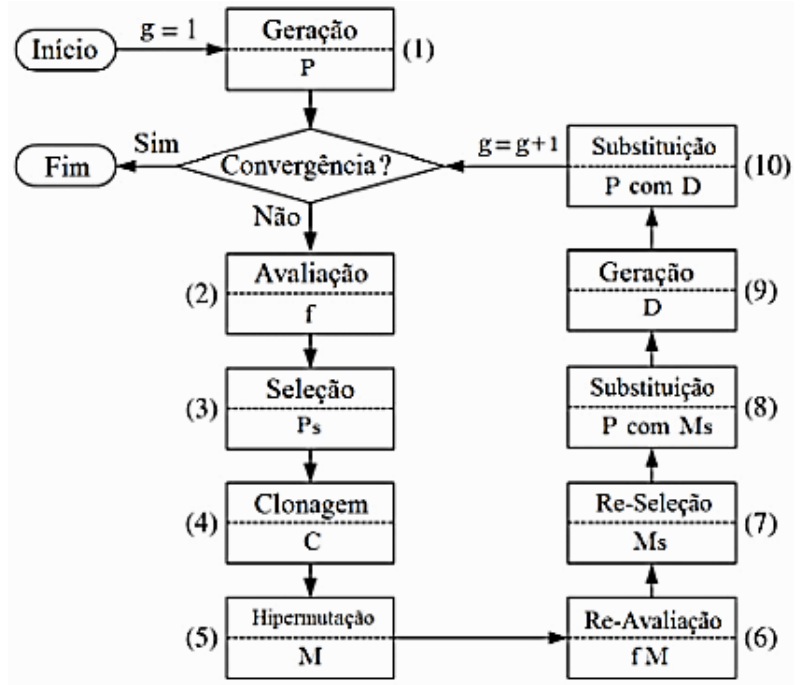

Figura 1. Algoritmo CLONR.

$\mathrm{Na}$ resolução dos exemplos propostos na Seção Aplicaçães, todos de minimização, foi adotado um padrão definido em dez passos:

1) São gerados 10 pontos aleatoriamente;

2) Analisa-se o comportamento da função objetivo para cada ponto;

3) São selecionados os 5 menores valores para função objetivo a ser minimizada, o restante é descartado.

4) Estes 5 melhores valores para minimização da função objetivo são clonados;

5) Os valores clonados são agora mutados. A mutação é dada em $x=x+0,01 x \operatorname{randn}(5,1)$, sendo $x$ um vetor 5 linhas por 1 coluna e randn valores aleatórios da distribuição normal, também 5 por 1 .

6) Avaliação dos 10 valores, clonados versus mutados.

7) Seleção dos 5 que mais minimizam a função objetivo. 

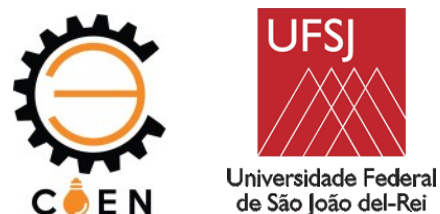

8,9) São gerados 10 pontos novamente e inseridos os 5 melhores resultantes da iteração anterior, obtidos no passo 7, gerando um vetor $x$, de dimensão 15 linhas por 1 coluna.

10) Analisa-se o comportamento da função objetivo, selecionando a melhor solução e avalia-se a convergência, neste caso adotada como 1000 iterações ou 100 iterações consecutivas sem melhora na solução superior a $10^{-8}$. Caso ocorra, este será o valor que mais minimiza a função objetivo, selecionado no passo 10. Caso contrário, quando não há convergência, volta-se ao passo 2 , com os 15 valores de $x$ indo para o próximo ciclo até que no passo três sejam descartados 10 valores, continuando normalmente o processo.

Na próxima Seção são apresentados exemplos para aplicação dos métodos, sendo a mais importante um estudo de caso de um Sistema Elétrico, seguindo o que vem sendo feito na literatura. Este sistema possui 3 barras e sua otimização consiste em minimizar o fluxo de potência ativa entre as linhas 2 e 3, sujeito à algumas restrições de tensão e potência. Na Subseção Resultados é feita a análise comparativa dos resultados obtidos para estes problemas através do Método de Newton e também pelo CLONR.

\section{APLICAÇÕES}

Foram feitas 3 aplicações para análise comparativa entre os métodos mencionados acima. Estes são apresentadas abaixo, separadamente de sua resolução que constará na Subseção Resultados.

A primeira aplicação é uma aplicação da Função de Rosenbrock, que é uma função muito usada em testes de algoritmos de otimização.

$$
\operatorname{Min} f(x, y)=(1-x)^{2}+100\left(y-x^{2}\right)^{2}
$$

O segundo problema, diz respeito a minimização de custos de produção de um paralelepípedo, com custos de materiais diferentes para cada superfície, volume e proporção entre largura e comprimento especificados.

$$
\left\{\begin{aligned}
& \text { Min } C=20(2 l w)+ 12(2 w h)+12(2 l h) \\
& \text { Sujeito } a: \quad l w h=100 \\
& l=3 w
\end{aligned}\right.
$$

O terceiro problema se trata da minimização do fluxo entre as linhas 2 e 3 de um sistema de três barras. As restrições deste problema são em relação à estabilidade de tensão e contra a motorização do gerador 1 , além dos balanceamentos de potência nas barras inerentes à problemas voltados ao sistema elétrico. Os dados do sistema são mostrados nas Tabelas 1 a 3 e as equações relativas ao problema na Equação 17:

$$
\left\{\begin{array}{r}
\text { Min } F_{23}=V_{2} V_{3}\left(g_{23} \cos _{23}+b_{23} \sin \theta_{23}\right) \\
0.95 p . u \leq V_{1}, V_{2}, V_{3} \leq 1.05 p . u \\
P g_{1}>0 \\
\text { Sujeito a } \\
\Delta P_{2}, \Delta P_{3}=0 \\
\Delta Q_{2}, \Delta Q_{3}=0
\end{array}\right.
$$


Tabela 1. Dados das linhas de transmissão.

\begin{tabular}{cccccc}
\hline Circ. & De & Para & Resist. & Reat. & S. shunt \\
\hline 1 & 1 & 2 & 0,042 & 0,042 & 0,041 \\
\hline 2 & 1 & 3 & 0,031 & 0,126 & 0,031 \\
\hline 3 & 2 & 3 & 0,031 & 0,126 & 0,031 \\
\hline
\end{tabular}

Tabela 2. Dados iniciais das barras do sistema.

\begin{tabular}{cccccc}
\hline Barra & Tipo & Tensão & Ang. & Pg & Qgmax \\
\hline 1 & SW & 1,04 & 0 & 300 & 150 \\
\hline 2 & PV & 1 & 0 & 180 & 150 \\
\hline 3 & PQ & 1 & 0 & 0 & 0 \\
\hline
\end{tabular}

Tabela 3. Dados das cargas do sistema.

\begin{tabular}{ccccc}
\hline Barra & Qgmin & Carga (PI) & Carga (QI) & Imp. shunt \\
\hline 1 & 0 & 65 & 30 & 0 \\
\hline 2 & 0 & 70 & 40 & 0 \\
\hline 3 & 0 & 115 & 60 & 0 \\
\hline
\end{tabular}

\subsection{Resultados}

Para simplificar as Tabelas, a função objetivo será chamada nas mesmas de Fobj. e as iterações até que seja atingido o critério de parada de Iconv.

i) O primeiro problema, representado pela Equação 15, não apresenta restrições e pode ser resolvido na sua forma literal. O resultado obtido via Método de Newton se encontra na Tabela 4, o resultado via CLONR na Tabela 5 e comparação percentual entre os resultados na Tabela 6 . As variáveis do Método de Newton são identificadas com o índice $n$ na Tabela 6 .

i-1) Aplicando o método de Newton ao primeiro problema proposto para uma tolerância de $10^{-8} \mathrm{e}$ chutes iniciais $x_{i}=20$ e $y_{i}=20$.

Tabela 4. Resultado do problema 1 por Newton.

\begin{tabular}{cccc}
\hline $\mathbf{x}$ & $\mathbf{y}$ & Fobj. & Iconv. \\
\hline 1,0000 & 1,0000 & 0,0000 & 5 \\
\hline
\end{tabular}

i-2) Aplicando agora a busca imunológica, para população inicial gerada $P=20 \operatorname{rand}(10,2)$.

Tabela 5. Resultado do problema 1 por busca imunológica.

\begin{tabular}{cccc}
$\mathbf{x}$ & $\mathbf{y}$ & Fobj. & Iconv. \\
\hline 1,0002 & 1,0006 & 0,0000 & 608 \\
\hline
\end{tabular}

Tabela 6. Comparação entre os resultados obtidos para o problema i).

\begin{tabular}{cccc}
\hline & $\left(\frac{\Delta x}{x_{n}}\right) 100 \%$ & $\left(\frac{\Delta y}{y_{n}}\right) 100 \%$ & $\left(\frac{\Delta f}{f_{n}}\right) 100 \%$ \\
\hline Valor (\%) & 0,020 & 0,060 & 0,000 \\
\hline
\end{tabular}



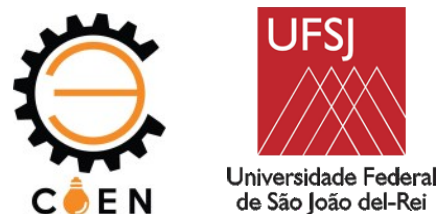

ii) O segundo problema, representado pela Equação 16, apresenta restrições de igualdade e deve ser reescrito observando a Equação 2, para resolução via Método de Newton, formando a Equação 18. O resultado obtido via Método de Newton se encontra na Tabela 7, o resultado via CLONR na Tabela 8 e comparação percentual entre os resultados na Tabela 9. As variáveis do Método de Newton são identificadas com o índice $n$ na Tabela 9.

$$
\operatorname{Min} C_{2}=20(2 l w)+12(2 w h+2 l h)+\lambda_{1}(l w h-100)+\lambda_{2}(l-3 w)
$$

ii-1) Foram atribuídos inicialmente, valores que não atendem as restrições para mostrar a convergência do método. Estes valores são: $l_{i}=10, w_{i}=10, h_{i}=10, \lambda_{1}=-10, \lambda_{2}=-10$.

Tabela 7. Resultado do problema 2 por Newton.

\begin{tabular}{ccccccc}
\hline $\mathbf{l}$ & $\mathbf{w}$ & $\mathbf{h}$ & $\boldsymbol{\lambda}_{\mathbf{1}}$ & $\boldsymbol{\lambda}_{\mathbf{2}}$ & Fobj. & Iconv. \\
\hline 7,1138 & 2,3713 & 5,9282 & $-13,4949$ & $-47,4252$ & 2024,2384 & 5 \\
\hline
\end{tabular}

ii-2) Para o algoritmo de busca imunológica, o problema pode ser reescrito conforme a Equação 14, como a Equação 19. A população inicial é gerada por $P=10 \operatorname{rand}(10,3)$.

$$
\text { Min } C_{3}=20(2 l w)+12(2 w h+2 l h)+w_{1}(l w h-100)+w_{2}(l-3 w)
$$

Contudo, a simulação não apresentou convergência para iteração menor ou igual a 1000, não atendendo o critério de convergência. Contudo, observou-se que ao aumentar o número de iterações permitidas para 10000 e aumentar o critério de estagnação para 1000, havia convergência. Neste caso, como os pesos tornam a função objetivo muito sensível a alterações nas variáveis, optou-se por criar um segundo critério de estagnação. Após a primeira convergência, à função de mutação é feita conforme a Equação 20. Esta foi por simplicidade apresentada para cada variável, no entanto, foi equacionada na forma matricial de forma a tornar o algoritmo mais eficiente. O critério do número de iterações das duas estagnações é cumulativo não podendo ultrapassar juntos os 10000 .

$$
\left\{\begin{array}{c}
l=l+0,001 l \text { randn }(5,1) \\
w=w+0,001 w \operatorname{randn}(5,1) \\
h=h+0,001 h \text { randn }(5,1)
\end{array}\right.
$$

A Tabela 8 apresenta os resultados obtidos na primeira e segunda convergências, sendo a primeira representada por $1^{\mathrm{a}}$ conv. e a segunda por $2^{\mathrm{a}}$ conv.

Tabela 8. Resultado do problema 2 por busca imunológica.

\begin{tabular}{cccccc}
\hline & $\mathbf{l}$ & $\mathbf{w}$ & $\mathbf{h}$ & Fobj. & Iconv. \\
\hline $\mathbf{1}^{\mathbf{a}}$ conv. & 7,0787 & 2,3621 & 5,9802 & 2024,4201 & 3360 \\
\hline $\mathbf{2}^{\mathbf{a}}$ conv. & 7,1103 & 2,3708 & 5,9319 & 2024,2592 & 2391 \\
\hline
\end{tabular}




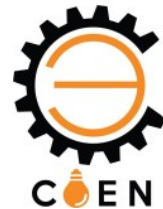

Tabela 9. Comparação entre os resultados obtidos para o problema ii).

\begin{tabular}{lcccc} 
& $\left(\frac{\Delta l}{l_{n}}\right) 100 \%$ & $\left(\frac{\Delta w}{w_{n}}\right) 100 \%$ & $\left(\frac{\Delta h}{h_{n}}\right) 100 \%$ & $\left(\frac{\Delta C}{C_{n}}\right) 100 \%$ \\
\hline $\mathbf{1}^{\mathbf{a}}$ conv. Valor (\%) & $-0,493 \%$ & $-0,388 \%$ & $0,877 \%$ & $0,009 \%$ \\
\hline $\mathbf{2}^{\mathbf{a}}$ conv. Valor (\%) & $-0,049 \%$ & $-0,021 \%$ & $0,062 \%$ & $0,001 \%$ \\
\hline
\end{tabular}

iii-1) Para o terceiro exemplo, aplicando a Equação 4 na Equação 17 para utilização do Método de Newton, as equações são reescritas conforme a Equação 21.

$$
\begin{aligned}
\operatorname{Min} F_{23}=V_{2} & V_{3}\left(g_{23} \cos _{23}+b_{23} \sin _{23}\right) \\
& +\lambda_{1}\left(\Delta P_{2}-0\right)+\lambda_{2}\left(\Delta P_{3}-0\right)+\lambda_{3}\left(\Delta Q_{2}-0\right)+\lambda_{4}\left(\Delta Q_{3}-0\right)+\mu_{1}\left(V_{1}\right. \\
& -0.95)+\mu_{2}\left(V_{1}-1.05\right)+\mu_{3}\left(V_{2}-0.95\right)+\mu_{4}\left(V_{2}-1.05\right)+\mu_{5}\left(V_{3}\right. \\
& -0.95)+\mu_{6}\left(V_{3}-1.05\right)+\mu_{7}\left(P g_{1}-0\right)
\end{aligned}
$$

Contudo, devido ao número de restrições e ao número elevado de zeros na matriz de derivadas segundas, $G$, o que dificulta e torna impreciso seu processo de inversão, a solução obtida para o problema não apresentou convergência, apresentando um resultado não ótimo.

iii-2) Para aplicação da busca imunológica, utilizando o método de penalização da Equação 14 e atribuindo o mesmo peso para todas as violações de restrição, o problema pode ser reescrito simplificadamente como a Equação 22.

$$
\begin{aligned}
\operatorname{Min} F_{23}=V_{2} & V_{3}\left(g_{23} \cos \Theta_{23}+b_{23} \sin \theta_{23}\right)+w\left(\left(\Delta P_{2}-0\right)+\left(\Delta P_{3}-0\right)+\left(\Delta Q_{2}-0\right)\right. \\
& \left.+\left(\Delta Q_{3}-0\right)+\left(V_{1}-0.95\right)+\left(V_{1}-1.05\right)\right)+\left(V_{2}-0.95\right)+\left(V_{2}-1.05\right) \\
& \left.+\left(V_{3}-0.95\right)+\left(V_{3}-1.05\right)+\left(P g_{1}-0\right)\right)
\end{aligned}
$$

Contudo, para este caso é usado conjuntamente o algoritmo de Newton-Raphson, muito utilizado em problemas de fluxo de potência, que garante que as restrições de potências da Equação 17 sejam atendidas, sendo sua formulação omitida e simplificando o problema. As exceções são estabilidade de tensão e motorização do gerador 1. O procedimento da busca imunológica é o mesmo descrito na seção Metodologia, sendo o algoritmo de Newton-Raphson responsável apenas por fazer o cálculo do estado do sistema para os valores de tensão e de potência gerada como variáveis $x$. A Equação 23 mostra a nova função a ser otimizada.

$$
\begin{array}{r}
\operatorname{Min} F_{23}=V_{2} V_{3}\left(g_{23} \cos \Theta_{23}+b_{23} \sin \theta_{23}\right)+w\left(\left(V_{1}-0.95\right)+\left(V_{1}-1.05\right)+\left(V_{2}\right.\right. \\
\left.-0.95)+\left(V_{2}-1.05\right)+\left(V_{3}-0.95\right)+\left(V_{3}-1.05\right)+\left(P g_{1}-0\right)\right)
\end{array}
$$

Desta forma o problema se reduz ao citado acima, em que são gerados a cada iteração $V_{1}, V_{2}$ e $P g_{2}$ aleatoriamente conforme mostrado na Equação 24. $V_{3}$ e $P g_{1}$ são resultados do algoritmo de Newton Raphson, que calcula o estado atual do sistema a partir desses dados de entrada e assumindo $\Theta_{1}=0$, como referência. 


$$
\left\{\begin{array}{c}
V_{1}=0.95+0.1 \operatorname{rand}(1,1) \\
V_{2}=0.95+0.1 \operatorname{rand}(1,1) \\
P g_{2}=180+0.02 \operatorname{randn}(1,1)
\end{array}\right.
$$

Se $V_{1}, V_{2}, V_{3}$, ou $P g_{1}$, violam as restrições impostas, $w=100$, caso contrário, $w=0$. Os resultados podem ser observados nas Tabelas 10 e 11. Resultado antes da otimização:

Tabela 10. Resultado do problema 3 antes da otimização.

\begin{tabular}{cccccc}
\hline $\boldsymbol{V}_{\mathbf{1}}$ & $\boldsymbol{V}_{\mathbf{2}}$ & $\boldsymbol{V}_{\mathbf{3}}$ & $\boldsymbol{P} \boldsymbol{g}_{\mathbf{1}}$ & $\boldsymbol{P}_{\mathbf{2}}$ & $\boldsymbol{F}_{\mathbf{2 3}}$ \\
\hline 1,0400 & 1,0000 & 0,9613 & 74,1255 & 180,0000 & 106,0811 \\
\hline
\end{tabular}

Resultados após a otimização:

Tabela 11.Resultado do problema 3 por busca imunológica com Newton Raphson.

\begin{tabular}{ccccccccc}
\hline $\boldsymbol{V}_{\mathbf{1}}$ & $\boldsymbol{V}_{\mathbf{2}}$ & $\boldsymbol{V}_{\mathbf{3}}$ & $\mathbf{I c o n v}$ & $\left.\boldsymbol{\Theta}_{\mathbf{2}} \mathbf{(}^{\mathbf{0}}\right)$ & $\left.\boldsymbol{\Theta}_{\mathbf{3}} \mathbf{(}^{\mathbf{0}}\right)$ & $\boldsymbol{P g}_{\mathbf{1}}$ & $\boldsymbol{P ~ g}_{\mathbf{2}}$ & $\boldsymbol{F}_{\mathbf{2 3}}$ \\
\hline 1,0494 & 0,9763 & 0,9509 & 588 & 8,9501 & 0,5163 & 0,5054 & 259,3537 & 67,1843 \\
\hline
\end{tabular}

Para os resultados do primeiro e segundo exemplos, o Método de Newton e a AIS mostraram resultados muito próximos, apesar de uma grande diferença no número de iterações. Na primeira aplicação as diferenças entre as variáveis foram inferiores à $0,06 \%$ e para a função objetivo os valores são iguais, se considerados até a quarta casa decimal. A segunda aplicação, apresentou para a primeira convergência, diferença entre as variáveis inferiores a $0,88 \%$ e entre as funções de custo aproximadamente $0,009 \%$. Na segunda convergência, as diferenças entre as variáveis foram menores ou iguais a $0,062 \%$ e entre as funções de custo aproximadamente $0,001 \%$.

O resultado da otimização do terceiro exemplo não apresentou convergência para o Método de Newton sozinho. Contudo, a combinação entre o Newton-Raphson e a Busca Imunológica conseguiu otimizar o problema, o que mostra que a combinação entre os métodos traz novas possibilidades de otimização. Neste exemplo, pode-se observar que para que haja redução no fluxo entre as linhas 2 e 3, o gerador 1 deverá operar no mínimo e o gerador 2 com potência maior, a tensão no gerador 1 deve ser elevada e a do gerador 2 reduzida, neste cenário, a tensão no gerador 3 fica próxima do limiar de 0,95 .

\section{CONCLUSÃO}

Uma revisão teórica sobre o método de Newton e o AIS através do método CLONR foi feita. Suas aplicações foram mostradas em três exemplos, em que os resultados obtidos apresentaram grande similaridade. Mesmo no pior caso, a diferença entre as variáveis foi menor do que $1 \%$ e entre as funções objetivo tendeu a zero, o que mostra que os métodos convergiram para o mesmo ponto, otimizando o problema. O algoritmo imunológico apresentou convergência mais lenta no caso de restrições de igualdade e o método de Newton apresenta problemas de convergência no caso de matriz de derivadas segundas próximas à singularidade, com muitas restrições. Para os problemas de restrição de desigualdade e para os problemas sem restrições o AIS apresentou um resultado muito próximo ao método de Newton, podendo ainda ser aplicado no terceiro caso onde este não se mostrou 

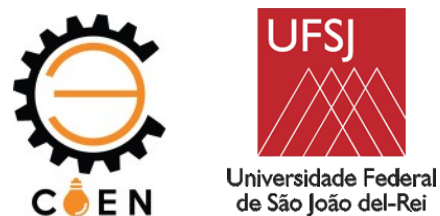

eficaz. A divisão do terceiro problema a dois algoritmos que trabalham em conjunto, sendo o de Newton responsável somente por estimar o estado do sistema, possibilitou que este fosse resolvido mais facilmente via busca imunológica. Isso mostra que a combinação entre métodos baseados em derivação e métodos baseados em meta-heurística é promissora para otimizações. Apesar da busca imunológica levar a um número maior de iterações, suas iterações são mais rápidas por não necessitarem do cálculo de derivadas e matrizes inversas em comparação com o método de Newton, apresentando tempo computacional pouco maior para os problemas apresentados. Com isso, o método mostrou sua utilidade prática nos exemplos aplicados, sendo uma ferramenta importante para análise de sistemas elétricos.

\section{REFERÊNCIAS}

BOUSSAÏD, Ilhem; LEPAGNOT, Julien; SIARRY, Patrick. A survey on optimization metaheuristics. Information Sciences, 2013, 237: 82-117.

COSSI, Antonio Marcos; LOPES, Mara Lucia Martins. Artificial Immune Systems Applied in Data Management Solutions to the Problem of Restoration of Electrical Distribution Systems. IEEE Latin America Transactions, 2016, 14.9: 4028-4034.

DE KLERK, E.; ROOS, C.; TERLAKY, T. Nonlinear Optimization (CO 367). 2004.

DE OLIVEIRA, Leonardo W. et al. Artificial immune systems applied to the reconfiguration of electrical power distribution networks for energy loss minimization. International Journal of Electrical Power \& Energy Systems, v. 56, p. 64-74, 2014.

FERREIRA, Ewerton Luiz; DE OLIVEIRA, Leonardo Willer; DE OLIVEIRA, Edimar José. Aplicação de Sistema Imunológico Artificial para Aumento da Estabilidade de Tensão de Sistemas de Distribuição, 2015.

LAU, Henry YK; LU, Steven YP. A Lagrangian based immune-inspired optimization framework for distributed systems. In: Systems, Man and Cybernetics, 2008. SMC 2008. IEEE International Conference on. IEEE, 2008. p. 1326-1331.

LIU, Bo; WANG, Ling; JIN, Yi-hui. Advances in differential evolution. Control and Decision, 2007, 22.7: 721 .

MATEI, Ion; BARAS, John S. Distributed nonlinear programming methods for optimization problems with inequality constraints. In: Decision and Control (CDC), 2015 IEEE 54th Annual Conference on. IEEE, 2015. p. 2649-2654.

NAVARRO-CACERES, Maria, et al. An Evaluation of a Metaheuristic Artificial Immune System for Household Energy Optimization. Complexity, 2018.

OLIVEIRA, Fabiana Rodrigues de, et al. Estudo de alguns métodos clássicos de otimização restrita não linear. 2012.

RIDZUAN, M. R. M., et al. Sustainable Environmental Economic Dispatch Optimization with Hybrid Metaheuristic Modification. Indonesian Journal of Electrical Engineering and Computer Science, 2018, 11.1: 161-168.

SAMPAIO, Phillipe Rodrigues. Teoria, métodos e aplicações de otimização multiobjetivo. 2011. PhD Thesis. Universidade de São Paulo. 

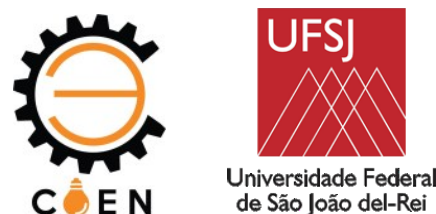

SANTOS, Turíbio José Gomes de. Um Novo Algoritmo de Penalização Hiperbólica para Resolução do Problema de Programação não-linear com Restrições de Igualdades, 1998.

SARAMAGO, Simone Pereira; STEFFEN JR, Valder. Introdução às técnicas de otimização em engenharia. Horizonte cientifico, 2008, 2.2.

SETA ${ }^{a}$, Felipe S., et al. Reconfiguração Ótima de Sistemas de Distribuição com Representação das Incertezas na Demanda através de Fluxo de Potência Intervalar, 2015.

SETA $^{\mathrm{b}}$, Felipe da Silva, et al. Reconfiguração de sistemas de distribuição considerando incertezas através de fluxo de potência intervalar e sistemas imunológicos artificiais, 2015.

SHIN, Joong-Rin, et al. A new optimal routing algorithm for loss minimization and voltage stability improvement in radial power systems. IEEE Transactions on Power Systems, 2007, 22.2: 648-657.

SILVA, Marcelo Araújo da. Aplicação do Lagrangeano aumentado em otimização estrutural com restrições dinâmicas. 1997. PhD Thesis. Universidade de São Paulo.

SINGH, Birender; CHAUHAN, Sushil; REDDY, C. C. Power loss minimization in electrical power distribution networks by use of hybrid reconfiguration method. In: Condition Assessment Techniques in Electrical Systems (CATCON), 2017 3rd International Conference on. IEEE, 2017. p. 349-354.

SWANN, W. H. A survey of non-linear optimization techniques. FEBS letters, 1969, 2.S1: S39S55.

TIAN, Hui-yuan, et al. An Extreme Learning Machine Based on Artificial Immune System. Computational Intelligence and Neuroscience, 2018.

ZEIDLER, Eberhard. Nonlinear functional analysis and its applications: III: variational methods and optimization. Springer Science \& Business Media, 2013.

\title{
COMPARATIVE STUDY OF NEWTON ALGORITM AND ARTIFICIAL IMMUNOLOGIC SYSTEMS AND APPLICATION ON TRANSMISSION LINE FLUX MINIMIZATION
}

\author{
Marcelo A. Canavez ${ }^{(1)}$ (marcelocanavez2@gmail.com), Jéssica B. Oliveira ${ }^{(2)}$ \\ (jessicabonoto@yahoo.com.br), Leônidas C.de Resende ${ }^{(3)}$ (leonidas@ufsj.edu.br) \\ (1,2) Programa de Pós-Graduação em Engenharia Elétrica (UFSJ), Praça Frei Orlando, 170 - Centro, São João del-Rei - MG, Brazil \\ (3) Universidade Federal de São João del-Rei (UFSJ) - DEPEL - Praça Frei Orlando, 170 - Centro, São João del-Rei - MG, Brazil
}

ABSTRACT: This paper presents a review of the optimization algorithm based on metaheuristic and inspired by Artificial Immune Systems (AIS), through the Clonal Selection with Reconfiguration Algorithm (CLONR). A brief contextualization and equation of the Newton Method as a derivation-based method is also presented. Three examples are presented with the goal of becoming a guide for development and resolution via Newton and via CLONR. Thus, the results obtained by the two algorithms can be compared in relation to the optimal point and the objective function value at this point. This document presents, among the applications, the minimization of power flow through a transmission line of a 3-bar system, to minimize the flow between two bars respecting the voltage and power limitations of the system and without motorizing the generator. The 
CLONR Method is strongly linked to the initial variable values and takes more iterations than Newton's Method. However, these iterations turn out to be too fast and can solve complex problems. This method, like others based on metaheuristics, does not have the obligation of the objective function to have derivatives of high orders, which happens with the Newton Method and others based on derivation.

KEYWORDS: Optimization, Artificial Immunologic System, CLONR, Power Flow, Newton, CLONALG. 